\title{
Grand challenges in molecular innate immunity
}

\section{Mohamed R. Daha*}

Department of Nephrology, Leiden University Medical Center, Leiden, Netherlands

${ }^{*}$ Correspondence: m.r.daha@lumc.nl

More than $95 \%$ of living organisms on the world protect themselves from infectious challenges using their innate immune system. The others protect themselves both by innate- and acquired mechanisms. The acquired immune system has been studied extensively and remains a focus for intensive investigation for the coming period. On the other hand there has also been a long history of investigation of certain components of innate immunity like the Toll and the complement system. These days we know that hosts like humans are optimally protected against pathogenic stress when both the innate and acquired immune systems are intact and functional.

In case of deficiencies or abnormalities in acquired immunity, like defective function of T- or B-cells and malfunction of innate immunity, like complement deficiencies, major disturbance of host protection may occur.

Several soluble and cellular mediator systems comprise innate immunity (Figure).

The soluble mediator systems amongst others are: cytokines, chemokines, defensins, pentraxins, the complement system, and presumably other, yet to define, mediator systems. The cellular components of innate immunity consist, amongst others, of NK cells, macrophages, granulocytes, eosinophils, and antigen presenting cells (dendritic cells). Upon entrance of pathogens almost instant recognition of the pathogens occurs by cellular and soluble pattern like recognition molecules. Concerning complement - mediated recognition, especially the recognition molecules like C1q, properdin, mannan binding lection, and Ficolins, play an important role.

Once recognition of a pathogen has occurred, complement activation and subsequent deposition of activated complement fragments, such as $\mathrm{C} 3 \mathrm{~b}$, takes place on the membrane of the pathogen which result in two major events. In the first place further activation of complement results in the generation of the membrane attack complex of complement and subsequent cytolysis. The other major function of complement activation is that pathogens opsonized with $\mathrm{C} 3 \mathrm{~b}$ or other breakdown fragments of C3 lead to engagement of cellular C3 receptors on many cells like macrophages, granulocytes, T-cells, B-cells, and antigen presenting cells. These interactions in the first place result in efficient elimination of foreign pathogens and additionally serve to present antigens in an optimal fashion, via cellular complement receptors, to antigen presenting cells and finally to the activation of T- and B-cells and induction of the acquired immune response. In this regard it is known for a long time that for instance
Innate and acquired immunity

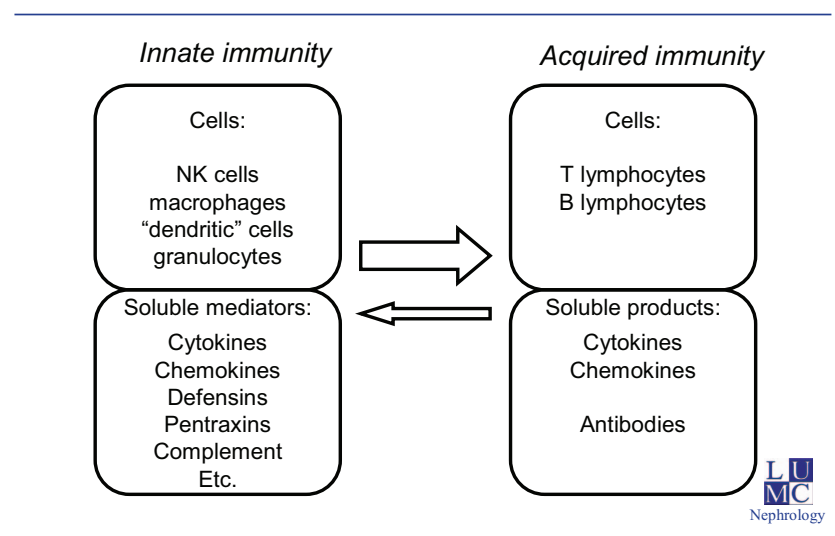

animals that are depleted of complement have a strongly reduced ability to mount an optimal acquired immune response to foreign antigens. Taken together complement acts as a rapid and efficient immune surveillance mechanism to control infection but also as an adjuvant for the induction of the acquired $\mathrm{T}$ - and B-cell response and ultimately in the generation of antibodies. Once acquired immunity is operational the products of acquired immunity such as antibodies interact with complement and other components of innate immunity and enhance protection against foreign pathogens.

There is also a downside to activation of the complement system as well. In the first place when tolerance against host tissue and cells is broken and auto-antibodies are induced, the complement system may react with these auto-antibodies, increase tissue injury and amplify disease progression. Additionally complement has been shown to recognize altered self, a condition that may occur upon injury of host cells for instance during oxygen deprivation. Once these modified tissues are recognized by the complement initiating molecules properdin, C1q, MBL, or Ficolins, other mediator systems like pentraxins may amplify complement activation and aggravate tissue injury. In general host cells are protected from complement-mediated injury by membrane bound regulators of complement activation. During tissue injury the expression of these regulator molecules may be reduced resulting in reduced protection of cells and tissue so that increased tissue damage can take place.

Recent studies have indicated that the balance between complement activation and control of activation is a delicate one. In cases where the turnover (activation) of C3 is enhanced, either in the circulation or at the tissue level, disease may take place as has been shown in patients with atypical hemolytic uremic syndrome, age related macular degeneration, and membranoproliferative glomerulonephritis. Therefore it 
seems essential to devise specific complement inhibiting therapies that can be used for the treatment of complement-mediated injuries.

The below bullet point comments give some examples of the big challenges faced by clinicians and basic scientists for the near future:

- Complement and complement receptor cross-talk: although major advance has been achieved in this field, like elucidation of the crystal structure of C3, major challenges lie ahead to define the many cellular receptor interactions and subsequent functions of several complement receptors.

- Induction of the acquired immune response: here the role of the several soluble mediator systems and their function in the initial recognition and presentation of antigens, via specific cellular receptors, to antigen presenting cells and T- and B-cells requires further major innovative approaches and finetuning. In this regard the cross-talk with toll like receptors has just started to be elucidated.

- How do chemokines and cytokines control the local production and function of complement: it is known that the major production site of complement components is the liver, however quite a number of complement components like properdin, $\mathrm{C} 1 \mathrm{q}$, and C7 are produced at extra hepatic sites as well. The contribution of local complement production and control by other tissues than the liver and their contribution to local defense and inflammation requires major attention.

- Control of innate and adaptive immunity: the cross-talk between innate immune players and products of acquired immunity in shaping an optimal defense against known and future infective challenges requires major attention. In this regard the use of the "adjuvant capacity" of complement to enhance the induction of the acquired immune response has to be exploited more optimally for the design of novel strategies and the development of next generation vaccines for known and yet to discover viral and bacterial organisms.

- Translational research: the generation of animals with deficiencies of several complement components and regulators has revealed that complement is not only involved in host defense but that it is a major player in tissue repair. Definition of the imbalance in local "homeostasis" in combination with complement abnormalities will be essential for the understanding of the molecular basis of several diseases where hyper catabolism of C3 takes place. In this regard the discovery and clinical implementation of innovative drugs and new biological reagents, to either attenuate or enhance complement activation, in specific diseases is essential.

These demanding objectives can only be reached through concerted efforts of clinicians and basic researchers.

A major challenge remains the translation of clinical observations to well defined research projects that can lead the way from basic discoveries to clinical implementation.

Received: 09 May 2011; accepted: 09 May 2011; published online: 31 May 2011.

Citation: Daha MR (2011) Grand challenges in molecular innate immunity. Front. Immun. 2:16. doi: 10.3389/ fimmu.2011.00016

This article was submitted to Frontiers in Molecular Innate Immunity, a specialty of Frontiers in Immunology.

Copyright (c) 2011 Daha. This is an open-access article subject to a non-exclusive license between the authors and Frontiers Media SA, which permits use, distribution and reproduction in other forums, provided the original authors and source are credited and other Frontiers conditions are complied with. 